\title{
Stable silencing of $\beta$-lactoglobulin (BLG) gene by lentivirus-mediated RNAi in goat fetal fibroblasts
}

\author{
Shumin Zhang*, Kai Xiong*, Zhourui Xie, Wenting Nan, Honglin Liu and Jie Chen \\ College of Animal Science and Technology, Nanjing Agricultural University, Nanjing, \\ People's Republic of China.
}

\begin{abstract}
$\beta$-lactoglobulin (BLG), a dominant allergen in goat milk, is difficult to remove by traditional biochemical methods. Its elimination from goat milk by genetic modification therefore poses a major challenge for modern goat breeders. $A$ shRNA targeting BLG mRNA with high interference efficiency was identified, with which lentiviral vectors were used for mediating stable shRNA interference in goat-fetal fibroblast cells. Apart from high efficiency in the knockdown of BLG expression in these cells, lentivector-mediated RNAi manifested stable integration into the goat genome itself. Consequently, an in vitro model for goat BLG-content control was compiled, and a goat-cell line for accompanying transgenetic goat production created.
\end{abstract}

Key words: RNAi, shRNA, lentivirus, BLG, goat.

Received: November 12, 2011; Accepted: February 4, 2012.

\section{Introduction}

Although nutritious for humans, especially infants, goat milk and its by-products are considered important allergens in food allergy, whence the restrictions against their wide use in the food industry. $\beta$-lactoglobulin (BLG), a major protein in milk (56\%-60\% of total cow-whey proteins), is considered a dominant allergen (Sharma et al., 2001; Fritsche, 2003).

Several biochemical approaches have been applied, in the attempt to reduce the allergenic potential of milk proteins, especially BLG. Heat treatment, enzymatic hydrolysis, fermentation and glycation (Fritsche, 2003; Hattori et al., 2004; Ehn et al., 2005), as well as the Maillard reaction (Guanhao Bu et al., 2010), are examples. Notwithstanding, these always entail increased costs and the accumulation of unexpected by-products. Hence, how to decrease BLG content in goat milk, and thus deaden milk allergy by genetic modification, remains a major challenge among modern goat-breeders.

RNA interference (RNAi) is now a widely-used technique for the sequence-specific knockdown of target mRNA (Dorsett and Tuschl, 2004; Mittal, 2004). The stable induction of RNAi usually involves short hairpin RNA (shRNA) expressed from plasmid or viral vectors. shRNA has a stem-loop structure that mimicks endogenous

Send correspondence to Jie Chen. College of Animal Science and Technology, Nanjing Agricultural University, 210095 Nanjing, People's Republic of China. E-mail jiechen @ njau.edu.cn.

*These authors contributed equally to this work as co-first authors.
microRNA (miRNA), besides sharing the same cleavage mechanism and transport pathways (McCaffrey et al., 2002; Miyagishi et al., 2004). In animal cells, mature 21-25 nt miRNAs are produced in cytoplasm, with incorporation of the antisense strand into the RNA-induced silencing complex (RISC), thereby mediating gene silencing, this including mRNA cleavage and translational repression. Lentiviral vectors are potent and efficient in enhancing the capacity for stable RNAi mediating-gene silencing in animal cells (Stewart et al., 2003; Xie et al., 2008). This capacity is critical for further application, especially gene knockdown in transgenic animals.

The aim was to obtain goat transgenic fetal fibroblasts with stable RNAi $B L G$-gene suppression. An in vitro cell model to mimic in vivo goat mammary epithelial cells was built by inducing $B L G$ overexpression in goat fetal fibroblasts. The model was then used to examine the efficiency of shRNA induced $B L G$ expression silencing, as well as the lentiviral vectors for mediating stable shRNA interference in goat fetal fibroblast cells.

\section{Materials and Methods}

\section{Construction of U6-shRNA plasmids}

The three shRNAs targeting goat $B L G$ and a negative control shRNA were designed by Dharmacon siDESIGN Center (Dharmacon, Waltham, USA), with mRNA sequences of goat $B L G(\mathrm{Z} 19569)$ (Table 1). The siRNAs were selected on a ranking criterion basis (Reynolds et al., 2004). The 5-nt loop (CAAGA) chosen for all the shRNAs was 
Table 1 - Oligonucelotides used in producing shRNA against goat BLG mRNA.

\begin{tabular}{ll}
\hline Oligos & Sequence \\
\hline shRNA1 & 5-CACCGGAGATCCTGCTGCAGAAATGTTCAAGAGACATTTCTGCAGCAGGATCTCCTTTTTTG-3 \\
& 3-CCTCTAGGACGACGTCTTTACAAGTTCTCTGTAAAGACGTCGTCCTAGAGGAAAAAACCTAG-5 \\
shRNA2 & 5-CACCGCGAGTGTGCTCAGAAGAAGATTCAAGAGATCTTCTTCTGAGCACACTCGCTTTTTTG-3 \\
& 3-CGCTCACACGAGTCTTCTTCTAAGTTCTCTAGAAGAAGACTCGTGTGAGCGAAAAAACCTAG-5 \\
shRNA3 & 5-CACCGAACGAGAACAAAGTCCTTGTTTCAAGAGAACAAGGACTTTGTTCTCGTTCTTTTTTG-3 \\
& 3-CTTGCTCTTGTTTCAGGAACAAAGTTCTCTTGTTCCTGAAACAAGAGCAAGAAAAAACCTAG-5 \\
Negative control & 5-CACCGTTCTCCGAACGTGTCACGTCAAGAGATTACGTGACACGTTCGGAGAACTTTTTTG-3 \\
shRNA & 3-CAAGAGGCTTGCACAGTGCAGTTCTCTAATGCACTGTGCAAGCCTCTTGAAAAAACCTAG-5 \\
\hline
\end{tabular}

then synthesized (GenePharma). Annealed oligonucleotides were ligated into pGP-U6 (GenePhrama) between the $B b s$ and Xho sites by T4 DNA ligase (TaKaRa), according to manufacturer's recommendations (Figure 1). Correct specific shRNA insertion was further confirmed by sequencing (Invitrogen).

\section{Construction of OE-BLG overexpression plasmids}

Total RNA was isolated from goat mammary gland tissues, whereupon reverse transcription was carried out to

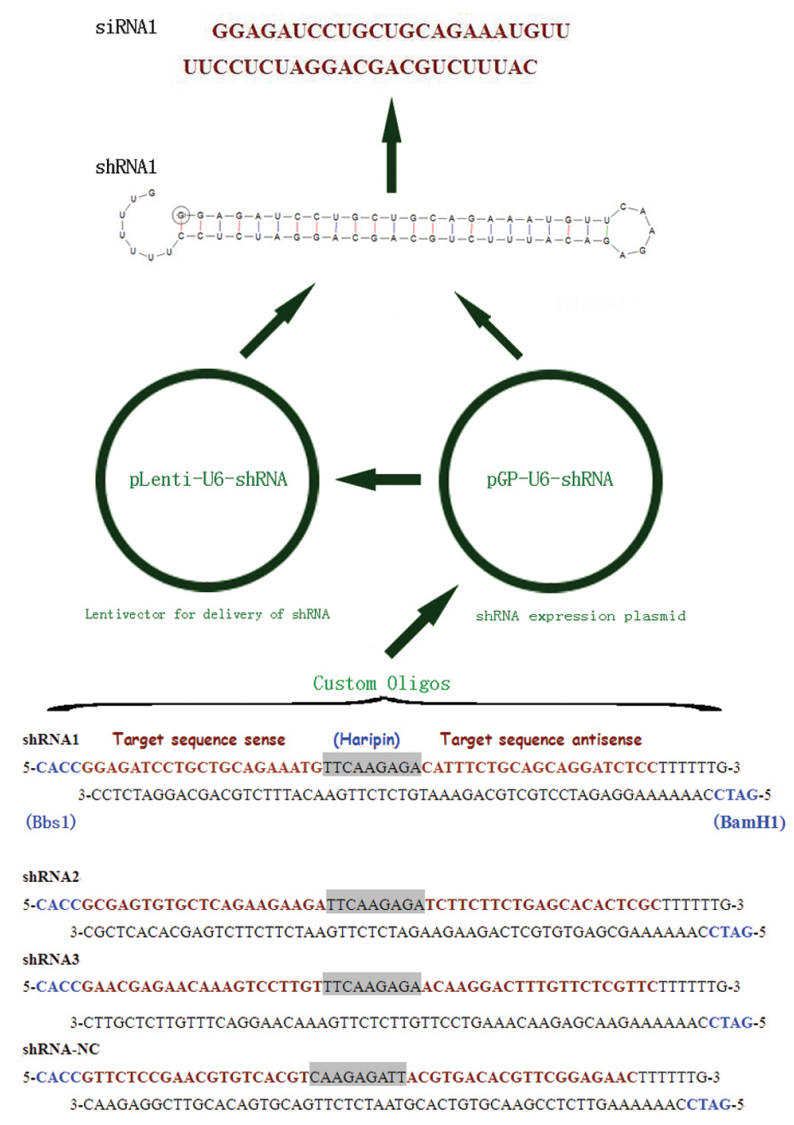

Figure 1 - Schematic illustration of shRNA expression and cleavage. Custom oligos of each shRNA were synthesized and ligated with plenti-LacZ fragment. shRNA expressed in stably transfected GEF cells. The shRNA hairpin structure is cleaved by the cellular machinery into siRNA, which is then bound to the RNA-induced silencing complex (RISC). This complex binds to and cleaves BLG mRNAs. prepare cDNA. A full-length wild-type goat $B L G$ open reading frame ( $758 \mathrm{bp}$ ) was obtained by PCR, using cDNA as templates, as well as forward (5'-GCACTCGAGAGA AGCACGA-3') and reverse (5'-GGCGGATCCCTTTA TTGCTGAAGGAGGA-3') primers. Xho and $B a m H$ restriction sites (underlined) were respectively flanked with initiation and stop codons by PCR. The resultant PCR products were digested with Xhoand $\mathrm{BamH}$, purified and cloned as overexpressional fragments into N1 vectors (Invitrogen). The final construct was termed OE- BLG.

\section{Construction of the lentivector for delivery of shRNA}

The lentivector backbone (6553 bp) was amplified by $\mathrm{PCR}$, using the pLenti6/V5-GW/LacZ vector (Invitrogen) as template, with Lenti-F (5'-CGA ATC GCG GAT CCG CGA CTT CCA GTT CAA-3') and Lenti-R (5'-GCA GGA CCA TCG ATC TCC ACC TTC TTC TTC TAT-3') as the respective forward and reverse primers. PCRs were according to the standard protocol for thermal stable PrimeSTAR HS DNA polymerase (TaKaRa). PCR products were separated by $1.0 \%$ agarose gel electrophoresis and stained with ethidium bromide (EB). The expected band was excised and recovered with the AxyPrep Gel Extraction Kit (AxyGene scientific, Inc). U6-shRNA2 cassettes (492 bp) were amplified by PCR, using as forward primer (5'-AAT TAA CCC TCA CTA AAG GG-3'), and reverse (5' -CCA TCG ATG TAA TAC GAC TCA CTA TAG GGC-3'). The resultant PCR products were then subcloned into the lentivector backbone by T4 ligase (Takara). The final construct was termed pLenti-U6- shRNA2 (Figure 1).

\section{Cell culture, transient transfection}

Goat embryo fibroblast (GEF) cells (donated by Prof. Feng Wang, Nanjing Agricultural University) were grown in Dulbecco's modified Eagle's minimal essential medium (DMEM)-F12 (Gibco) supplemented with 15\% fetal bovine serum (FBS) (Gibco) at $37^{\circ} \mathrm{C}$, and $5 \%$ carbon dioxide $\left(\mathrm{CO}_{2}\right) .24 \mathrm{~h}$ before transfection, $3 \times 10^{5}$ cells were seeded into each well of a 6-well plate (Costar), and cultured in the growth medium without antibiotics, to so achieve $90 \%$ $95 \%$ confluence at the appropriate time. For transfection, $4 \mu \mathrm{g}$ of each shRNA expression construct was used per 
well. Lipofectamine 2000 Reagent (Invitrogen) was used as the transfection reagent, according to manufacturer's instructions, i.e., mingling $4 \mu \mathrm{g}$ of DNA with $6 \mu \mathrm{L}$ of lipofectamine per well. For co-transfection, each shRNA construct was co-transfected with N1-BLG (2:1) into GEF cell cultures using lipofectamine. After $6 \mathrm{~h}$ past transfection, the lipofectamine \& DNA mixture was removed from the wells and replaced by a fresh medium. After $48 \mathrm{~h}$ past transfection, cells were assayed by quantitative RT-PCR, with non-transfected and non-shRNA vector transfected cells as controls. 293FT virus producer cells (Invitrogen) were cultured in a complete D-MEM medium containing $10 \%$ FBS supplemented with $0.1 \mathrm{mM}$ MEM Non-Essential Amino Acids, $1 \mathrm{mM}$ sodium pyruvate and $2 \mathrm{mM}$ Lglutamine. $293 \mathrm{FT}$ cells were maintained in the complete medium containing $500 \mu \mathrm{g} / \mathrm{mL}$ Geneticin (Invitrogen).

\section{Real-time RT-PCR analysis}

Total RNA was isolated $48 \mathrm{~h}$ after transfection using Trizol (Invitrogen) according to manufacturer's instructions. All RNA samples were reverse transcribed in a $25 \mu \mathrm{L}$ reaction mixture at $37^{\circ} \mathrm{C}$ for $15 \mathrm{~min}$ with $M-M L V$ reverse transcriptase (Promega), using Oligo-dT. The cDNA samples were stored at $-20^{\circ} \mathrm{C}$, prior to quantification and analysis. Primers were designed for $B L G$ (Z19569) which span intron(s) to prevent amplification of genomic DNA, using the forward primer (5'-GGC GAG TGT GCT CAG AAG A-3') and the reverse primer(5'-GGG CTC AGC ACT GTT TTC C-3'). Real-Time RT-PCR (ABI 7300) was performed using FastStart Universal SYBR Green Master (Roche), according to manufacturer's protocol. PCR thermal cycle reactions were denaturation at $95^{\circ} \mathrm{C}$ for $10 \mathrm{~min}$ followed by 40 cycles of denaturation and annealing/extension at $95{ }^{\circ} \mathrm{C}$ for $15 \mathrm{~s}$ and $60{ }^{\circ} \mathrm{C}$ for $1 \mathrm{~min}$. Amplification specificity during real-time RT-PCR was monitored by evaluating the melting curve and examining the products of real-time RT-PCR on agarose gel for the absence of nonspecific bands. The comparative $\mathrm{Ct}$ (threshold cycle) method (Livak and Schmittgen, 2001) was used to determine the relative amount of RNA transcripts of BLG gene present in the cells. The expression levels of each group of cells were normalized to $\beta$-actin levels, using the forward primer (5'-TGA ACC CCA AAG CCA ACC-3') and the reverse primer (5'-AGA GGG GT A CAG GGA CAG CA-3').

\section{Production of lentivirus in 293FT cells}

For the generation of lentivirus, 293FT cells were transfected with $9 \mu \mathrm{g}$ of ViraPower Packaging mix (Invitrogen) along with $5 \mu \mathrm{g}$ of pLenti-U6-shRNA2 plasmid by lipofectamine. $24 \mathrm{~h}$ post transfection, the transfection mix was replaced by a fresh culture medium (without antibiotics). The virus-containing supernatant was harvested $72 \mathrm{~h}$ post transfection, cleared by centrifugation $(3000 \mathrm{rpm} / \mathrm{min}$,
$15 \mathrm{~min}$, and $4^{\circ} \mathrm{C}$ ), and then filtered through a $0.45 \mu \mathrm{m}$ filter (Millipore). Lentivirus aliquots were subsequently prepared and stored at $-80^{\circ} \mathrm{C}$, until use in transduction. Virus generation and infection of target cells were carried out in class II biological safety cabinets in class II containment laboratories, with access restricted to authorized staff.

\section{GEF-cell transduction with recombinant lentivirus and selected by Blasticidin}

The day before transduction, goat embryo fibroblast cells were seeded into 6-well cell-culture plates at a density of $5 \times 10^{4}$ cells per well. On the day, they were transduced with $1 \mathrm{~mL}$ of the already described virus-containing supernatant. At $24 \mathrm{~h}$ post transduction, the mix was replaced with fresh cell-culture medium. $48 \mathrm{~h}$ post transduction, the cells were collected for RNA isolation and qRT-PCR analysis.

In order to produce stably transduced cells $48 \mathrm{~h}$ post transduction, $5 \mu \mathrm{g} / \mathrm{mL}$ of Blasticidin (Invitrogen) were added to the medium (DMEM/F12 + 15\% FBS), as a means of selecting clones containing the insert. The blasticidincontaining medium was replenished every 3 days. The cells remained in the selective medium for 2 weeks, after which they were left to grow to $70 \%$ confluence before RNA isolation and qRT-PCR analysis. 2-fold serial dilutions of Blasticidin were prepared in a preliminary test, $5 \mu \mathrm{g} / \mathrm{mL}$ of Blasticidin being considered as the final selective concentration. After 12 days, Blasticidin-resistant GEF cell pools were established.

\section{Statistical analysis}

Real-time PCR results were analyzed by using the $2^{-\Delta \Delta C T}$ method (Livak and Schmittgen, 2001). All the experiments were performed in triplicate. Data were analyzed with the SPSS 13.0 Student $t$-test (SPSS Inc., Chicago, IL) to evaluate the significance of differences in gene expression. The results were expressed as mean \pm STD. The level chosen to define whether treatments were significantly different from mock controls was $\mathrm{p}<0.05$.

\section{Results}

\section{shRNAs induce transient silencing of BLG in GEF}

Individual shRNA efficiency in inducing RNAi in GEF cells was tested in transiently transfected cells. Three shRNA constructs against $B L G$ were examined (Figure 1). As EGF cells have no de novo $B L G$ gene expression under the in vitro culture system, an in vitro model was established to investigate $B L G$ RNAi, by subcloning the fulllength cDNA fragment of goat $B L G$ gene into an overexpression plasmid (OE-BLG), and co-transfecting with shRNA expression plasmids. Synchronous with OE-BLG plasmid co-transfection, all the anti-BLG shRNA constructs resulted in a significant reduction $(\mathrm{p}<0.05)$ in the level of $B L G$ mRNA, compared to cells that were only transfected with OE-BLG plasmids, considered as positive 
control (Figure 2). As expected, as negative control, a luciferase-specific shRNA (shRNA-NC) failed to reduce $B L G$ mRNA levels, thereby showing no significant difference from cells that were only transfected with OE-BLG. All told, the interference efficiency that shRNAs induced in GEF cells was acknowledged, and the specialty of shRNA targeting confirmed.

Out of the three shRNA constructs, shRNA2 presented the highest efficiency in silencing $B L G$ expression in GEF cells, with a reduction of approximately $90 \%$, when compared with the positive control, whereas in the other two, although significantly high, this was less than $75 \%$ (Figure 2). Thus, shRNA2 was chosen for sequential establishment of BLG-knockdown goat-cell lines.

\section{Lentivirus-mediated shRNAs integrate into goat genome and provide stable BLG knockdown in GEF}

The shRNA2 expression cassette was removed from pGP-U6-shRNA plasmid by restriction digestion, for posterior subcloning into a lentivector system, newly denominated "plenti-U6-shRNA2". On using these plasmids together with a virus packaging mix, shRNA2-recombined lentiviruses were produced for infecting GEF cells. After remaining 12-days in the final Blasticidin-containing selective medium, surviving GEF colonies were defined, whereas control cells without lentivirus infection had expired, thereby demonstrating the establishment of a BLGknockdown GEF cell-line.

To confirm the integration of lentivector-mediated shRNA into cell-line genome, genome DNA was isolated from BLG-knockdown GEF cells for detection of shRNA expression cassettes. Through analysis with agarose gel

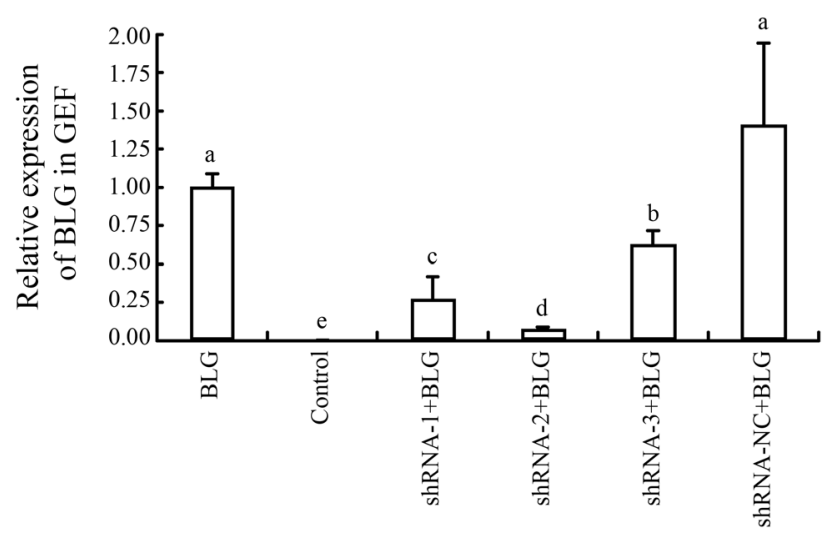

Figure 2 - Detection of shRNA efficiency against BLG. BLG mRNA levels were relatively lower in GEF cells co-transfected with both shRNA and BLG overexpression plasmids (2:1). Relatively higher BLG mRNA levels indicate those co-transfected with shRNA-NC and BLG overexpression plasmids (2:1), and those only transfected with BLG overexpression plasmids. shRNA-2 manifested the highest efficiency in BLG expression silencing. The amount of BLG mRNA overexpression was determined using Real-Time RT-PCR and normalized to $\beta$-actin mRNA. All the experiments were done in triplicate. The different letters indicate a significant difference of relative levels in each group $(\mathrm{p}<0.05)$ and error bars the s. d.. Control, non-transfected GEF cells. electrophoresis, these transgenetic fragments were detected in PCR products, but not in non-virus-infected control GEF cells (Figure 3). Further confirmation by gel extraction and gene sequencing provided additional evidence of, shRNA-expression-cassette stable integration into the goat genome.

In order to test BLG RNAi in established BLGknockdown GEF, OE-BLG was transfected into this cell line, while using normal GEFs as control. After $48 \mathrm{~h}$ of transfection, the mRNA was isolated and relative levels of $B L G$ in each group of GEFs analyzed. Significantly, the high efficiency of RNAi against BLG in BLG-knockdown GEFs was observed, whereas in control GEFs, $B L G$ expression remained at a relatively higher level $(\mathrm{p}<0.01)$ (Figure 4). All told, these data confirmed the establishment of BLG-knockdown GEFs with the ability to express de novo shRNA against BLG.

\section{shRNA interference efficiency correlates with the secondary structure of target mRNA}

Among all the shRNA constructs, shRNA2 presented the highest interference efficiency (Figure 2). This difference in efficiency can be explained by the interaction between target mRNA and complement RNA produced by shRNA. To investigate whether the $B L G$ mRNA secondary structure could affect RNAi interference efficiency, this structure was calculated at $37^{\circ} \mathrm{C}$. The calculated image showed shRNA1 and shRNA3 target regions to be selfparing in a large part and folded in stem structure (Figure 5). On the contrary, the shRNA2 target region was shown to unfold and stretch in a loop structure (Figure 5). Such diversity in mRNA secondary structure indicated variable

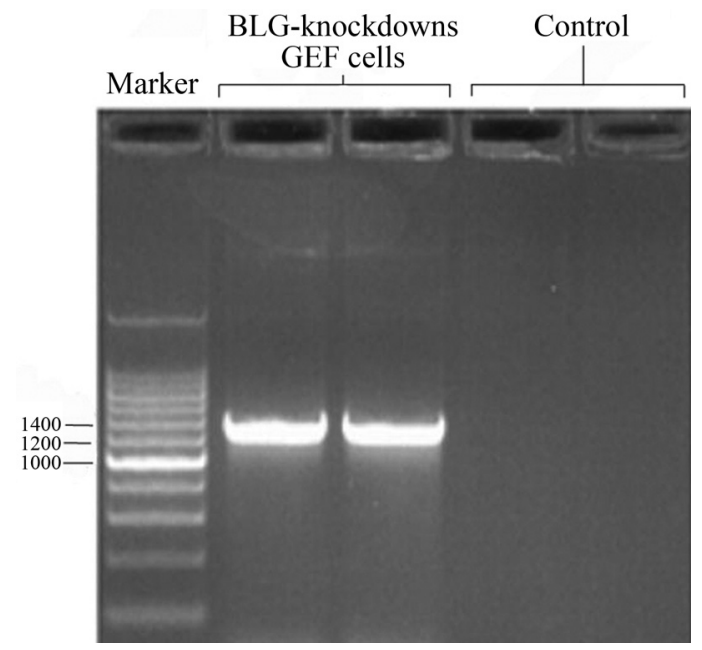

Figure 3 - Lentivector-mediated shRNA were stably integrated into the goat genome. In order to construct BLG-knockdown GEF cells, GEF cells were transfected with lentivector-mediated shRNA. After 15-days of selection with Blasticidin, GEF genomic DNA was isolated for plentiLacz-shRNA2 PCR identification. PCR products were analyzed by $1 \%$ agarose gel electrophoresis. The electrophoresis image indicates lentivector-mediated shRNA as being stably integrated into the goat genome. Control, non-infected GEF cells. 


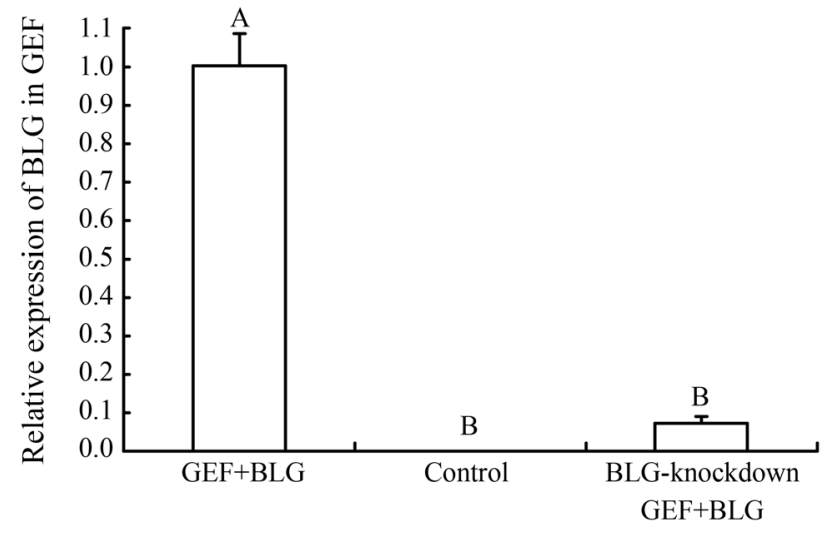

Figure 4 - Lentivector-mediated shRNAs silence BLG expression in BLG-knockdown GEF cells. BLG-knockdown GEF cells stably transfected with the lentivector-mediated shRNA construct demonstrated high efficiency in BLG expression silencing. The amount of BLG mRNA was determined using Real-Time RT-PCR and normalized to $\beta$-actin mRNA. Each group was detected in triplicate. The different capital letters indicate the highly significant difference of relative levels in each group $(p<0.01)$. Error bars indicate the sd.. Control, non-transfected GEF cells

molecular behavior while complement-RNA targeting, this resulting in differences in interference efficiency.

\section{Discussion}

In the present study, BLG expression silencing was with the lentivirus system mediating a stable RNAi in goat embryo fibroblasts. Lentivirus-mediated shRNA expression leads to long-term ability in silencing $B L G$ expression. In the established BLG-knockdown GEFs, induced $B L G$ expression was suppressed to $10 \%$ lower than in positive control cells (Figure 4). Such high efficiency had also been found in former shRNA transient transfection experiments (Figure 2). All together, the data indicate the capacity of the lentivirus system to effectively integrate sufficient DNA copies into goat genome to thus greatly deaden BLG expression, even in the disappearance of the interference caused by transient transfection.

Due to growing popularity, RNA-interference has become widely used in innumerous cases of gene silencing. Through induction by plasmids or virus vectors, RNAi has broadened its range of application (Bartel, 2004). DNA vectors facilitate the delivery of shRNA expression constructs into animal cells, to so achieve continuous longterm expression from either polor pol promoters, such as U6 (Brummelkamp et al., 2002). Besides infecting a wide range of cells in vitro and in vivo (Naldini et al., 1996), the lentivirus system is notably effective in shRNA delivery (Abbas-Terki et al., 2002). This system can also provide higher integration efficiency, whereas with conventional protocols depending on homologous recombination, integration efficiency was much lower, i.e., about $10^{-6}$. Even compared to the classical DNA microinjection (DNA-MI) technique, the system results in a four to eight-fold higher rate of transgenic animals per embryo treated (Pfeifer, 2004). In all these applications, nearly all the $\mathrm{F}_{0}$ generation animals expressed the transgene (Lois et al., 2002; Pfeifer et al., 2002). Although not with goats, lentiviral transgenesis has been applied with high efficiency in producing trangenic large farm animals, such as pigs and cattle (Hofmann et al., 2003, 2004; Whitelaw et al., 2004). Hence, by using established BLG-knockdown GEF, our research work on transgenic goat production can be further developed.

shRNA2 interference efficiency is the highest in transient transfection expression. The assumption that $B L G$ mRNA secondary structure may affect interference efficiency was amply sustained through subsequent computer calculations. As shRNA1 and shRNA3 target regions appeared over a large part of self-paring and folds in stem

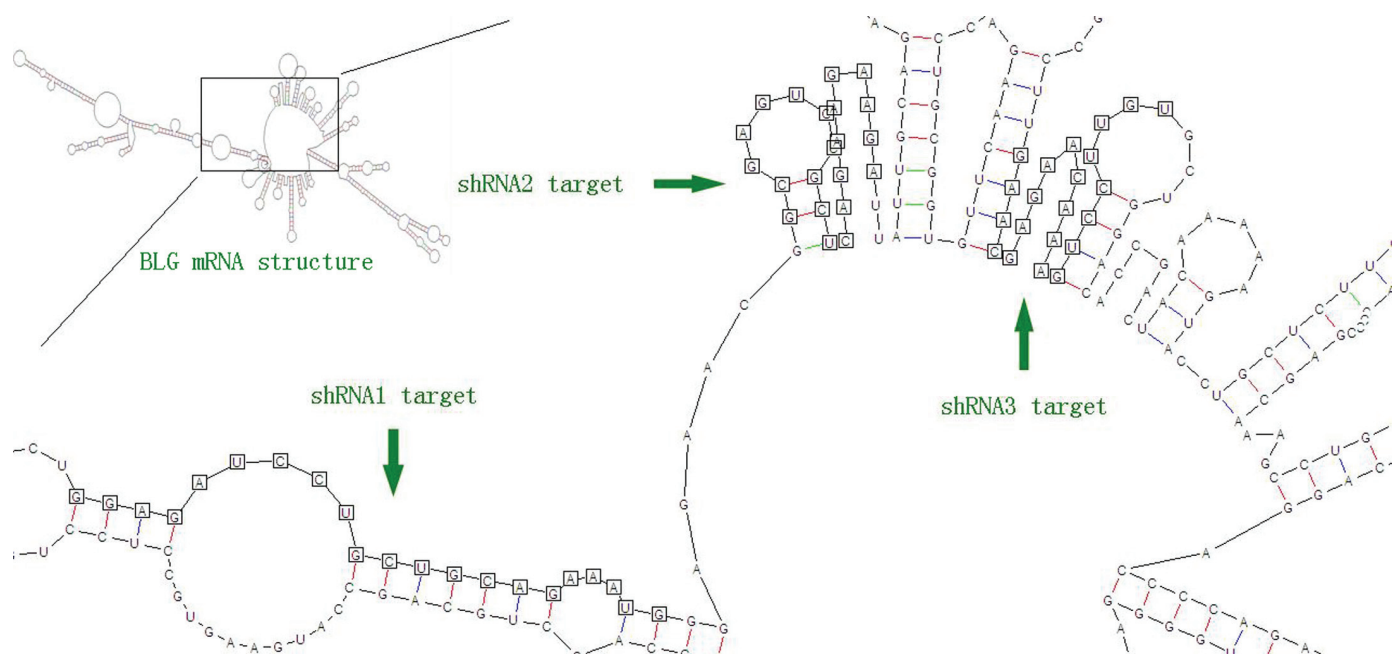

Figure 5 - shRNA target regions in BLG mRNA. BLG mRNA secondary structure at $37{ }^{\circ} \mathrm{C}$ was calculated to analyze mRNA target structural effects on RNAi efficiency. shRNA1 and shRNA3 target region structures were self-paring and stem-like, whereas the shRNA2 target region was relatively unfolded and looped. 
structures, these could hardly unfold for complementary RNA guiding RISC complex binding and function. On the contrary, as the shRNA2 target region showed unfolding and stretching in loop structures, this secondary structure is apparently more feasible for targeting proceedures (Shao et al., 2007), thereby displaying higher interference efficiency in silencing $B L G$ expression, whereby the assumption that secondary structure analysis of target mRNA is a credible method for predicting shRNA interference efficiency.

In conclusion, we are the first to use the RNAi mechanism via the lentivirus system to knockdown goat $B L G$ expression. Our shRNAs successfully target BLG mRNA and reduce expression to a low level. Compared to the conventional protocol depending on homologous recombination, the letivirus system can provide higher integration efficiency, thus demonstrating its potencial as an efficient tool for transgenetic application. In addition, the establishment of BLG-knockdown GEF can be of use for further genetic engineering to produce transgenic goats.

\section{Acknowledgments}

This work is financially supported by the China National Program for Transgenic Animal (08ZX08008-004).

\section{References}

Abbas-Terki T, Blanco-Bose W, Deglon N, Pralong W and Aebischer P (2002) Lentiviral mediated RNA interference. Hum Gene Ther 13:2197-2201.

Bartel DP (2004) MicroRNAs: Genomics, biogenesis, mechanism, and function. Cell 116:281-297.

Brummelkamp TR, Bernards R and Agami R (2002) A system for stable expression of short interfering RNAs in mammalian cells. Science 296:550.

Dorsett Y and Tuschl T (2004) siRNAs: Applications in functional genomics and potential as therapeutics. Nat Rev Drug Discov 3:318-329.

Ehn BM, Allmere T, Telemo E, Bengtsson U and Ekstrand B (2005) Modification of IgE binding to b-lactoglobulin by fermentation and proteolysis of cow's milk. J Agr Food Chem 53:3743-3748.

Fritsche R (2003) Role of technology in dairy allergy. Aust J Dairy Technol 58:89-91.

Hattori M, Miyakawa S, Ohama Y, Kawamura H, Yoshida T, To-o K, Kuriki T and Takahashi K (2004) Reduced immunogenicity of b-lactoglobulin by conjugation with acidic oligosaccharides. J Agr Food Chem 52:4546-4553.

Hofmann A, Kessler B, Ewerling S, Weppert M, Vogg B, Ludwig H, Stojkovic M, Boelhauve M, Brem G and Wolf E (2003) Efficient transgenesis in farm animals by lentiviral vectors. EMBO Rep 4:1054-1058.

Hofmann A, Zakhartchenko V, Weppert M, Sebald H, Wenigerkind H, Brem G, Wolf E and Pfeifer A (2004) Generation of transgenic cattle by lentiviral gene transfer into oocytes. Biol Reprod 71:405-409.

Bu G, Luo Y, Lu J and Zhang Y (2010) Reduced antigenicity of b-lactoglobulin by conjugation with glucose through controlled Maillard reaction conditions. Food Agr Immunol 21:143-156.

Livak KJ and Schmittgen TD (2001) Analysis of relative gene expression data using real-time quantitative PCR and the $2^{-\Delta \Delta \mathrm{CT}}$ method. Methods 25:402-408.

Lois C, Hong EJ, Pease S, Brown EJ and Baltimore D (2002) Germline transmission and tissue-specific expression of transgenes delivered by lentiviral vectors. Science 295:868-872.

Mittal V (2004) Improving the efficiency of RNA interference in mammals. Nat Rev Genet 5:355-365.

McCaffrey AP, Meuse L, Pham TT, Conklin DS, Hannon GJ and Kay MA (2002) RNA interference in adult mice. Nature 418:38-39.

Miyagishi M, Sumimoto H, Miyoshi H, Kawakami Y and Taira K (2004) Optimization of an siRNA-expression system with an improved hairpin and its significant suppressive effects in mammalian cells. J Gene Med 6:715-723.

Naldini L, Blomer U, Gallay P, Ory D, Mulligan R, Gage FH, Verma IM and Trono D (1996) In vivo gene delivery and stable transduction of nondividing cells by a lentiviral vector. Science 272:263-267.

Pfeifer A, Ikawa M, Dayn Y and Verma IM (2002) Transgenesis by lentiviral vectors: Lack of gene silencing in mammalian embryonic stem cells and preimplantation embryos. Proc Natl Acad Sci USA 99:2140-2145.

Pfeifer A (2004) Lentiviral transgenesis. Transgen Res 13:513522.

Reynolds A, Leake D, Boese Q, Scaringe S, Marshall WS and Khvorova A (2004) Rational siRNA design for RNA interference. Nat Biotechnol 22:326-330.

ShaoY, Chan CY, Maliyekkel A, Lawrence CE, Roninson I and Ding Y (2007) Effect of target secondary structure on RNAi efficiency. RNA 13:1631-1640.

Sharma S, Pravindra K, Betzel C and Singh TP (2001) Structure and function of proteins involved in milk allergies. J Chromatogr B 756:183-187.

Stewart SA, Dykxhoorn DM, Palliser D, Mizuno H, Yu EY, An DS, Sabatin DM, Chen IS, Hahn WC, Sharp PA, et al. (2003) Lentivirus-delivered stable gene silencing by RNAi in primary cells. RNA 9:493-501.

Whitelaw C, Radcliffe PA, Ritchie WA, Carlisle A, Ellard FM, Pena RN, Rowe J, Clark AJ, King TJ and Mitrophanous KA (2004) Efficient generation of transgenic pigs using equine infectious anaemia virus (EIAV) derived vector. FEBS Lett 571:233-236.

Xie S, Fang W, Liu Z, Wang S, Li X, Liu T, Xie W and Yao K (2008) Lentivirus-mediated RNAi silencing targeting ABCC2 increasing the sensitivity of a human nasopharyngeal carcinoma cell line against cisplatin. J Translat Med $6: 55$

Associate Editor: Carlos F.M. Menck

License information: This is an open-access article distributed under the terms of the Creative Commons Attribution License, which permits unrestricted use, distribution, and reproduction in any medium, provided the original work is properly cited. 